EPJ Web of Conferences 81, 05016 (2014)

DOI: $10.1051 /$ epjconf/ 20148105016

(C) Owned by the authors, published by EDP Sciences, 2014

\title{
Simple trigonometric proof for the new parameters of the sigma meson
}

\author{
Robert Kamiński ${ }^{1, a}$ \\ ${ }^{1}$ Henryk Niewodniczański Institute of Nuclear Physics, Polish Academy of Sciences, 31-342, Kraków, Poland
}

\begin{abstract}
Correctness of the results obtained in the recent analysis of the $\pi \pi$ interactions using new dispersion relations with imposed crossing symmetry condition is checked. The performed proof is based on purely mathematical relations and properties of analytic functions and concerns position of the scalar-isoscalar $f_{0}(500)$ (former $\sigma$ ) pole. Mere analysis of amplitudes expressed by the trigonometric functions and their derivatives clearly defines the area in which mass of the $\sigma$ and its width must be located.
\end{abstract}

\section{Method and results}

Results on the $\sigma$ pole position presented in [1] have been obtained using dispersive analysis without any model assumptions about specific energy dependence of the $\pi \pi$ amplitudes in the $S(S 0)$ and $P$ waves [2].

To prove uniqueness of the new position of the $\sigma$ pole one can use results of analysis presented in [3]. The authors fitted so called "Old" amplitudes with heavy and very wide $\sigma$ meson (see [4]) to the experimental data and to dispersion relations with imposed crossing symmetry condition i.e. to GKPY equations taken from [2]. They obtained "New" amplitudes and observed significant shift of the $\sigma$ pole to position almost overlapping with that found in [1]. Phase shifts corresponding to both of these amplitudes are presented on Fig. 1. Noticeable is significant differences between them especially between 500 and $800 \mathrm{MeV}$. One should also notice their completely different curvatures in this region caused by very different positions of the $\sigma$ pole. Figure 1 presents also energy dependence of the $K T_{\ell \ell^{\prime}}^{I I^{\prime}}=K T_{00}^{00}$ being dominant term in the GKPY equations for the scalar-isoscalar partial wave (for details see [2]). Its shape is completely given by crossing symmetry condition.

Real parts of the "Old" $S 0$ input and output amplitude in the GKPY equations are presented on the Fig. 2. In order to diminish difference between them one could intuitively think on a shifting down and up the real part of the input amplitude below and above about $650 \mathrm{MeV}$ respectively. As is seen in the Fig. 3 it would lead to decrease of the phase shifts below about $900 \mathrm{MeV}$ and simultaneously would reduce the imaginary part of the amplitude being an input in the GKPY equations. Comparison of the gradients of the imaginary and real parts seen on this figure shows that just the imaginary part changes faster than the real one.

Taking into account those facts and the shape of the dominant part of the GKPY equations shown on Fig. 1, defined almost entirely by crossing symmetry condition, one can conclude that mentioned

a e-mail: robert.kaminski@ifj.edu.pl 

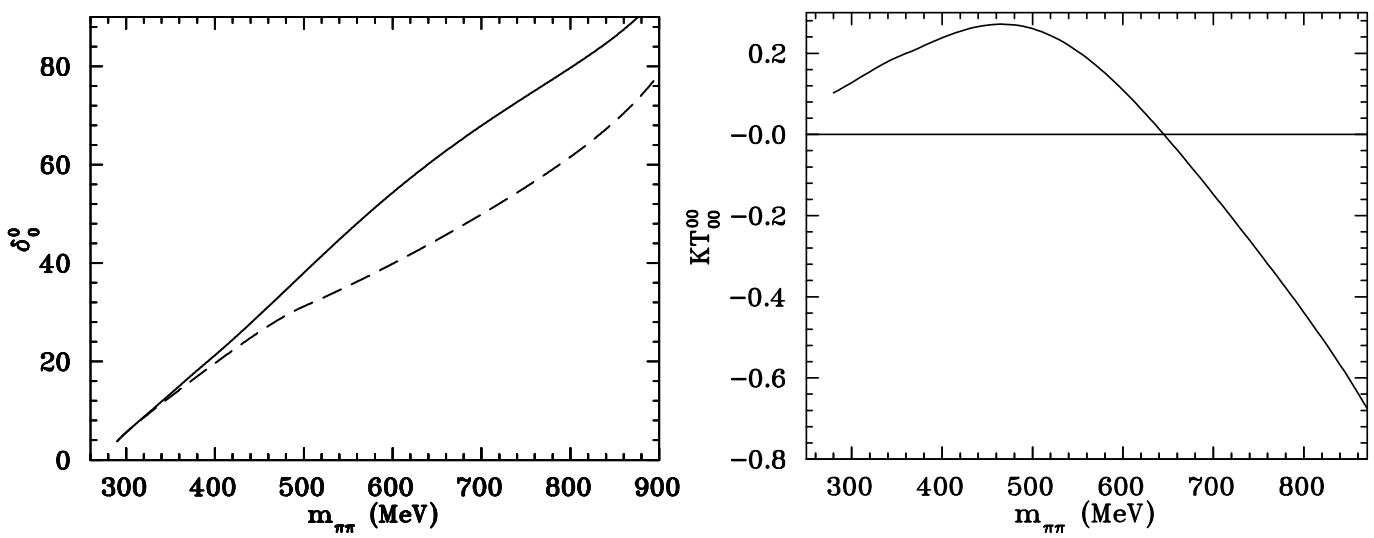

Figure 1. Left: effective two-pion mass dependence of the phase shifts corresponding to the old (dashed line) and new i.e. re-fitted (solid line) amplitudes. Right: effective two-pion mass dependence of the leading part of the GKPY equations.
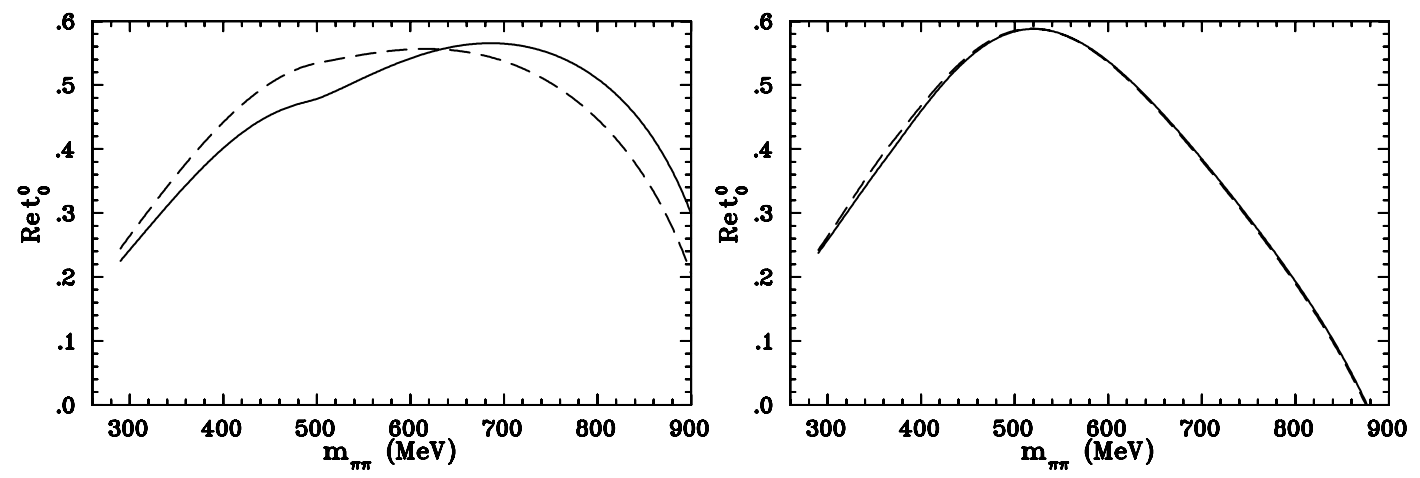

Figure 2. Input (dashed lines) and output (solid lines) real parts of the $S 0$ wave for amplitudes before fitting left panel and for amplitudes after fitting - right panel.

above decrease of the phase shifts would cause faster decrease of the output amplitude than decrease of the real part of the input one below around $650 \mathrm{MeV}$ and faster increase above this energy. It means that the input amplitude would not catch up the escaping output one. Therefore in order to diminish distance between them the phase shifts must not decrease but increase below about $800 \mathrm{MeV}$. Then, finally, the real parts of the input and output amplitudes can almost overlap (see Fig. 2) indicating that crossing symmetry condition below around $1 \mathrm{GeV}$ is fulfilled.

\section{Conclusions}

This mathematical proof shows that the increase of the phase shifts and change of the curvature seen on the Fig. 1, is unique consequence of the simple trigonometry relations and of the energy dependence of leading part of the GKPY equations given by the crossing symmetry condition. Such modification 
MESON $2014-13^{\text {th }}$ International Workshop on Production, Properties and Interaction of Mesons
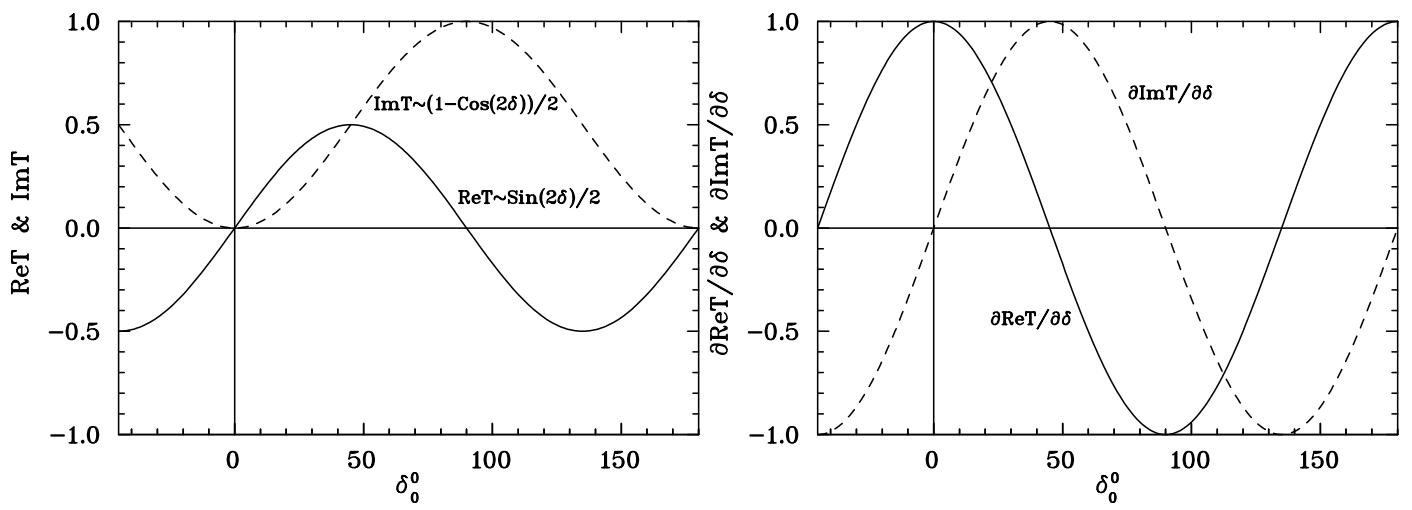

Figure 3. Left figure: real and imaginary part of the amplitude as a functions of the phase shifts, right figure: gradient of the real and imaginary part of the amplitude.

of the phase shifts can be produced only by the $\sigma$ pole on the IInd Riemann sheet lying closer to the physical and the imaginary axis than previously i.e. by narrower and lighter $\sigma$ meson.

Such position of the $\sigma$ pole agrees very well with results of another analysis [5] based on twice subtracted dispersion relations with imposed crossing symmetry condition i.e. so called Roy's equations. This position is $441_{-8}^{+16}-272_{-13}^{+9} \mathrm{MeV}$ while in analysis [1] is $457_{-13}^{+14}-279_{-7}^{+11} \mathrm{MeV}$. It is worth noting these completely independent analyses gave also similar errors of the pole position and were using different methods in determining the $S$ and $P$ wave $\pi \pi$ amplitudes below $800 \mathrm{MeV}$.

\section{Acknowledgements}

This work has been funded by the Polish National Science Center (NCN) grant DEC-2013/09/B/ST2/04382.

\section{References}

[1] R. Garcia-Martin, R. Kamiński, J. R. Pelaez and J. Ruiz de Elvira, Phys. Rev. Lett. 107 (2011) 072001

[2] R. Garcia-Martin, R. Kamiński, J. R. Pelaez, J. Ruiz de Elvira and F.J. Yndurain, Phys. Rev. D 83, 074004 (2011).

[3] V. Nazari, P. Bydžovský, R. Kamiński, Acta Phys. Pol. B45 (2014) 7, 1549.

[4] Yu. S. Surovtsev, P. Bydžovský, R. Kamiński and M. Nagy, Phys. Rev. D 81, 016001 (2010).

[5] I. Caprini, G. Colangelo, H. Leutwyler Phys. Rev. Lett. 96 (2006) 132001. 